\title{
Driving an improved CAR for cancer immunotherapy
}

\author{
Xiaopei Huang ${ }^{1}$ and Yiping Yang ${ }^{1,2}$ \\ 'Division of Hematologic Malignancies and Cellular Therapy, Department of Medicine, and 2Department of Immunology, Duke University, Durham, North Carolina, USA.
}

\begin{abstract}
The recent clinical success of chimeric antigen receptor (CAR) T cell therapy for B cell malignancies represents a paradigm shift in cancer immunotherapy. Unfortunately, application of CAR T cell-mediated therapy for solid tumors has so far been disappointing, and the reasons for this poor response in solid tumors remain unknown. In this issue of the $\mathrm{JCl}$, Cherkassky and colleagues report on their use of a murine model of human pleural mesothelioma to explore potential factors that limit CAR $T$ cell efficacy. Their studies have uncovered the importance of the tumor microenvironment in the inhibition of CAR T cell functions, revealed a critical role for the programmed death-1 (PD-1) pathway in CAR T cell exhaustion within the tumor microenvironment, and demonstrated improved antitumor effects with a CAR T cell-intrinsic PD-1 blockade strategy using a dominant negative form of PD-1. Together, the results of this study lay the groundwork for further evaluation of mechanisms underlying CAR T cell immune evasion within the tumor microenvironment for the improvement of CAR T cellmediated therapy for solid tumors.
\end{abstract}

\section{CAR T cells for cancer immunotherapy}

$\mathrm{T}$ cells that have been genetically engineered to express a chimeric antigen receptor (CAR) have emerged as one of the most promising therapeutic approaches for cancer (1). Along with immune checkpoint blockade (2), CAR T cell therapy has triggered a paradigm shift in cancer immunotherapy. This groundbreaking advance has also inspired the biotech and pharmaceutical industry to invest in and develop CAR $\mathrm{T}$ cell therapies for various types of cancer.

CAR technology was first developed in the mid-1980s (3) and later modified by the Eshhar group to use a single-chain variable fragment $(\mathrm{scFv})$ derived from both heavy and light chains of a monoclonal antibody $(4,5)$. The prototype CAR is composed of an extracellular scFv domain, which serves as the binding region for specific tumor-associated antigens (TAAs), a transmembrane domain, and an intracellular signaling domain derived from the
$\mathrm{T}$ cell receptor (TCR), usually the $\mathrm{CD} 3 \zeta$ chain, which triggers $\mathrm{T}$ cell activation (Figure 1). Most CAR-encoding constructs are introduced into $\mathrm{T}$ cells via plasmid or mRNA transfection or by viral vector transduction. These CAR T cells recognize unprocessed cell-surface antigens directly and destroy tumor cells independently of major histocompatibility complex (MHC). This recognition process is in contrast with that of conventional $\mathrm{T}$ cells, which rely on their native TCRs for recognition of tumor antigens presented by MHC. The composition of first-generation CARs involves the $\mathrm{CD} 3 \zeta$ intracellular signaling domain alone (Figure 1). Second- and third-generation CARs add one and two costimulatory domains, respectively, to the $\mathrm{CD} 3 \zeta$ intracellular regions, such as CD28 and CD137 (4-1BB), in an effort to augment the proliferation and persistence of CAR T cells (6) Second-generation CAR $\mathrm{T}$ cells that specifically target the B cell-specific antigen CD19 have shown impressive clinical out-

Related Article: p. 3130

Conflict of interest: The authors have declared that no conflict of interest exists

Reference information: J Clin Invest. 2016;126(8):2795-2798. doi:10.1172/JCI88959.

comes in treating patients with relapsed or refractory $\mathrm{B}$ cell malignancies, including acute and chronic lymphocytic leukemia (7-9), with a complete remission (CR) rate of more than $80 \%$ to $90 \%$ achieved in patients with acute lymphocytic leukemia. As such, the United States Food and Drug Administration (FDA) has recently granted the "breakthrough therapy" designation to the anti-CD19 CAR T cell therapy (10).

A growing number of CAR $\mathrm{T}$ cells designed to target solid tumors have been evaluated in clinical trials; however, the results so far have been modest (11). The trials using anti-diganglioside GD2 CAR $\mathrm{T}$ cells for neuroblastoma and anti-HER2 CAR to target HER2 ${ }^{+}$sarcoma are considered to be the most positive so far. An anti-GD2 CAR $\mathrm{T}$ cell trial yielded a CR rate of $27 \%$ (3 of 11 patients) (12), and an anti-HER2 CAR T cell trial showed 4 of 17 patients with stable disease (13). The reason CAR $\mathrm{T}$ cells do not result in a robust response in solid tumors remains to be elucidated. Multiple barriers have been suggested, including suboptimal TAAs for CAR $\mathrm{T}$ cell targeting, an inability of CAR T cells to traffic to and infiltrate solid tumors, and the immunosuppressive tumor microenvironment (11). It has been well recognized that even after successful trafficking and infiltration of $\mathrm{T}$ cells into growing tumors, these tumor-infiltrating lymphocytes (TILs) are often ineffective at tumor elimination in vivo, but are able to proliferate and exert effector functions when removed from the immunosuppressive environment. Within the tumor microenvironment, TILs must overcome function-suppressing challenges including immune suppressor cells, such as Tregs, myeloid-derived suppressor cells (MDSCs), and tumor-associated macrophages (TAMs); the presence of cytokines and soluble factors associated with immunosuppression, such as TGF- $\beta$ and IL-10; and T cell-intrinsic, negative regulatory mechanisms, such as upregulation of ligands for the inhibitory receptor programmed death-1 (PD-1) that downmodulate $\mathrm{T}$ cell activity (14). Thus, the same immunosuppressive microenvironment 


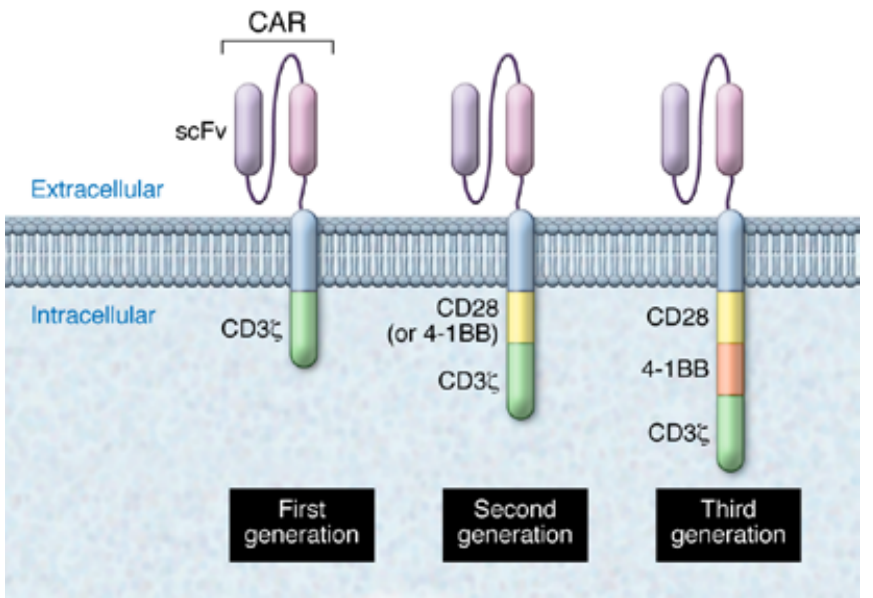

Figure 1. CAR T cell design. All CARs contain an extracellular antigen-binding domain derived from an ScFv that is composed of both the heavy and light chains of a TAA-targeting monoclonal antibody and an intracellular-signaling domain that is usually derived from the TCR CD3 $\zeta$ chain to activate $T$ cells. Only the $\mathrm{CD} 3 \zeta$ signaling domain is present in first-generation CARs. For second-generation CARs, one additional costimulatory domain, such as CD28 or 4-1BB, is added, while third-generation CARs contain two costimulatory domains.

that inhibits the activity of endogenous TILs might also prevent CAR T cells from generating effective antitumor responses.

\section{CAR T cells with immune checkpoint blockade}

A better understanding of the immunesuppression mechanisms within the tumor microenvironment has led to the recent success of immune checkpoint therapy with antibodies that block cytotoxic T-lymphocyte-associated protein 4 (CTLA-4) and PD-1 pathways in cancer patients (2). Blockade of PD-1 and its ligand PD-L1 is currently among the most promising therapies in clinical oncology. Antibodies that target the PD-1 pathway have been approved by the FDA for the treatment of solid tumors, such as melanoma and nonsmall cell lung cancer, and ongoing efforts to expand the indication for the treatment of other solid tumors and Hodgkin's lymphoma are underway (15).

Recent studies have indicated that infused CAR T cells express PD-1 and, therefore, are susceptible to PD-1-mediated suppression $(16,17)$. An obvious next step is to explore whether concurrent immune checkpoint blockade improves the outcome of CAR T cell therapies in solid tumors. An initial proof-of-concept study has already shown that blocking PD-1 immunosuppression can enhance the efficacy of CAR $\mathrm{T}$ cell therapy in a murine model of $\mathrm{HER}_{2}{ }^{+}$tumors, where adoptive transfer of anti-HER2 CAR T cells in combination with PD-1 blockade led to marked tumor regression (18). In this issue, Cherkassky and colleagues report on the effect $\mathrm{PD}-1-$ mediated $\mathrm{T}$ cell exhaustion has on anti-mesothelin CAR $\mathrm{T}$ cells and the generation of CAR $\mathrm{T}$ cells devoid of PD-1 to explore a cell-intrinsic checkpoint blockade strategy for improving the outcome of CAR T cell therapy in a xenograft model of human pleural mesothelioma in immunodeficient (NSG) mice (19). The authors performed a comprehensive analysis of the effector functions of intrapleurally infused CAR T cells in their model and have convincingly shown that second-generation $\mathrm{CD} 28-\mathrm{CD} 3 \zeta$ or 4-1BB-CD3ל CAR T cells are subject to suppression of effector functions, as cytolytic activity and cytokine secretion were inhibited in these cells upon tumor antigen encounter in vivo. Interestingly, although CAR $T$ cell persistence was enhanced to a similar degree by the inclusion of either 4-1BB or CD28 costimulatory signals, only

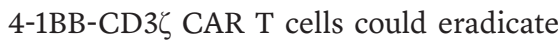
tumors at a lower $\mathrm{T}$ cell dose. The ability of $4-1 B B-C D 3 \zeta$ CAR T cells to function at a lower dose was accompanied by relative resistance to exhaustion within the tumor microenvironment. Moreover, Cherkassky and colleagues have shown that PD-1 ligands on tumor cells and PD-1 on the infused CAR T cells are upregulated in vivo, with a relatively higher level of
PD-1 expression observed on CD28-CD3ל CAR T cells. Furthermore, PD-1 antibody blockade restored the effector function of exhausted CD28-CD3 $\zeta$ CAR T cells in vitro and in vivo. Having shown a critical role of PD-1 signaling in CAR T cell exhaustion in vivo, Cherkassky and colleagues went on to generate CD28-CD3 C CAR T cells that express a dominant negative form of PD-1 (CD28-CD3ל-dnPD-1), and infusion of CD28-CD3 $\zeta$-dnPD-1 CAR T cells into tumor-bearing mice led to functional persistence, superior antitumor effect, and prolonged survival.

The data presented by Cherkassky and colleagues have demonstrated the importance of tumor-mediated inhibition of CAR $\mathrm{T}$ cell effector functions, revealed a critical role for the PD-1 pathway in CAR T cell exhaustion within the solid tumor microenvironment, and enabled the authors to provided an exciting and translatable strategy that involves using dnPD-1 CAR T cells alone or CAR T cells with PD- 1 antibody blockade in an effort to improve the outcome of CAR T cell-mediated therapy for solid tumors. There are some limitations with this study that should be considered. First, the use of a xenograft tumor model in immunocompromised NSG mice, which lack endogenous T cells and NK cells, may prevent a comprehensive evaluation for the contribution of other immunosuppressive mechanisms, such as Tregs, within the tumor microenvironment. In addition, it should be noted that these immunodeficient mice are not adequate to evaluate potential toxicities associated with CAR T cell infusion, as they lack pivotal immune cells and effector molecules. Thus, when such a model system is used to illustrate the CAR T cell response against human tumors, results should be interpreted with caution and these important findings will need to be confirmed in immunocompetent mice, ideally in a model of endogenously arising tumors. Second, the mechanism or mechanisms for inhibition of CAR T cell function may vary with different tumors, given the complexity of the tumor microenvironment. Thus, additional tumor models will be required to assess the generalizability of the PD-1 pathway in suppressing CAR T cell function and to identify other potential mechanisms of immune evasion. Indeed, the majority of mice treated with CD28-

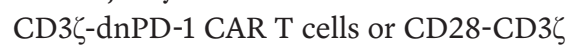


CAR T cells in combination with PD-1 antibody blockade eventually died of tumor progression, suggesting the presence of other inhibitory pathways/mechanisms. Finally, the observation that $4-1 \mathrm{BB}-\mathrm{CD} 3 \zeta$ CAR $\mathrm{T}$ cells are relatively resistant to $\mathrm{T}$ cell exhaustion within the tumor microenvironment is highly informative and may have a substantial impact on CAR $\mathrm{T}$ cell design. It remains unknown how 4-1BB costimulation confers resistance to CAR T cell exhaustion. Previous studies in nonCAR T systems have shown that systemic 4-1BB activation induces a potent $\mathrm{T}$ cell function that is driven by a high expression of eomesodermin (Eomes) in a model of melanoma (20). Additionally, the transcriptional factor $\mathrm{T}$-bet sustains $\mathrm{CD}^{+} \mathrm{T}$ cell responses during chronic infection by repressing PD-1 expression (21). Interestingly, Cherkassky et al. also observed that 4-1BB-mediated resistance associates with a lower level of PD-1 expression and a T-bet ${ }^{\text {hi }}$ Eomes ${ }^{\text {hi }}$ PD- ${ }^{\text {lo-like }}{ }^{-}$phenotype. Thus, it is possible that $4-1 \mathrm{BB}-\mathrm{CD} 3 \zeta$ CAR T cells repress PD-1 through Eomes. Further studies are needed to delineate the underlying mechanisms responsible for $4-1 \mathrm{BB}-$ mediated resistance in CAR T cell exhaustion within the tumor microenvironment.

\section{Clinical implications and future directions}

One of the main hurdles for the clinical translation of CAR T cell therapies for solid tumors has been the immunosuppressive tumor microenvironment. Cherkassky and colleagues have now demonstrated a critical role of PD-1 signaling in CAR T cell exhaustion within the tumor microenvironment and have provided a translatable approach that combines CAR T cell therapy and PD-1 blockade, either systemically with the anti-PD-1/anti-PD-L1 or locally with cell-intrinsic blockade in dnPD-1 CAR $\mathrm{T}$ cells. While both approaches are effective in augmenting the efficacy of CD28CD3 $\zeta$ CAR T cells, the use of dnPD-1 CAR $\mathrm{T}$ cells may reduce toxicity profile associated with systemic injection of anti-PD-1 or anti-PD-L1 antibodies and avoid the need for repeated antibody administration. However, additional safety features are necessary to avoid potentially augmented "on-target/off-tumor" toxicities associated with dnPD-1 CAR T cells. Suicide gene systems have been used in clinical trials to eliminate $\mathrm{T}$ cells with the administration of a small molecule inducer or prodrug, such as iCaspase-9 (22) or herpes simplex virus thymidine kinase (23). In fact, there is already an ongoing clinical trial with antimesothelin CD28-CD3C CAR T cells that have the iCaspase-9 safety switch incorporated (ClinicalTrials.gov NCT02414269). Thus, similar safety strategies can be introduced into CD28-CD35-dnPD-1 CAR T cells before evaluation in clinical trials.

Although the study by Cherkassky and colleagues provides an improved CAR to tackle poor responses by CAR T cells in solid tumors, more work is needed to understand other mechanisms responsible for the suppression of CAR T cell function within the tumor microenvironment. Given the diversity and complexity of the solid tumor microenvironment, it is possible that different tumors may employ different mechanisms for their immune evasion. Besides PD-1, other inhibitory pathways, such as CTLA-4, lymphocyte-activation gene 3 (LAG-3), $\mathrm{T}$ cell membrane protein 3 (TIM-3), and $\mathrm{T}$ cell immunoreceptor with Ig and ITIM domains (TIGIT), may also inhibit CAR T cell function. Similarly, investigation of immune suppressors, such as MDSCs, TAMs and Tregs, may show that these cells also blunt CAR T cell activity within the tumor microenvironment. Although Cherkassky et al. employed pleural delivery of CAR $\mathrm{T}$ cells, alternative delivery strategies to improve CAR T cell trafficking to the tumor site after systemic infusion should be explored. Finally, the identification/design of suitable specific target antigen may also be critical for the success of CAR $\mathrm{T}$ cell therapy in solid tumors. Unlike with CD19-CARs for B cell malignancies, it has been difficult to identify specific target antigens on solid tumors. A fatal on-target/off-tumor pulmonary toxicity in a colon cancer patient treated with anti-HER2 CAR T cells has been attributed to low-level expression of HER2 on lung epithelial cells (24). As improved CARs, such as dnPD-1 CAR, are developed, this potential on-target/off-tumor toxicity may be increased. One way to prevent this type of toxicity is to insert suicide genes, such as iCaspase-9 (22). Another approach to increase tumor specificity of CARs is to engineer "dual-specificity" CARs that require recognition of two antigens to achieve killing activity (25).
In summary, the work by Cherkassky and colleagues represents an exciting development in the CAR T cell field. Continued efforts to improve our understanding of how CAR T cell function is suppressed within the tumor microenvironment will provide important insights into the design of more effective CAR $\mathrm{T}$ cell therapies for solid tumors.

\section{Acknowledgments}

This work was supported by NIH grants (to Y.Y.) R01 CA136934, R01 CA193167, and R21 CA186973.

Address correspondence to: Yiping Yang, Department of Medicine, Duke University Medical Center, Box 103005, Durham, North Carolina 27710, USA. Phone: 919.668.0932; E-mail: yang0029@ mc.duke.edu.

1. Jackson HJ, Rafiq S, Brentjens RJ. Driving CAR T-cells forward. Nat Rev Clin Oncol. 2016;13(6):370-383.

2. Topalian SL, Drake CG, Pardoll DM. Immune checkpoint blockade: a common denominator approach to cancer therapy. Cancer Cell. 2015;27(4):450-461.

3. Gross G, Waks T, Eshhar Z. Expression of immunoglobulin-T-cell receptor chimeric molecules as functional receptors with antibody-type specificity. Proc Natl Acad Sci U S A. 1989;86(24):10024-10028.

4. Eshhar Z, Waks T, Gross G, Schindler DG. Specific activation and targeting of cytotoxic lymphocytes through chimeric single chains consisting of antibody-binding domains and the $\gamma$ or $\zeta$ subunits of the immunoglobulin and T-cell receptors. Proc Natl Acad Sci US A. 1993;90(2):720-724.

5. Stancovski I, Schindler DG, Waks T, Yarden Y, Sela M, Eshhar Z. Targeting of T lymphocytes to Neu/HER2-expressing cells using chimeric single chain Fv receptors. JImmunol. 1993;151(11):6577-6582.

6. Geldres C, Savoldo B, Dotti G. Chimeric antigen receptor-redirected $\mathrm{T}$ cells return to the bench. Semin Immunol. 2016;28(1):3-9.

7. Porter DL, Levine BL, Kalos M, Bagg A, June $\mathrm{CH}$. Chimeric antigen receptor-modified T cells in chronic lymphoid leukemia. $N$ Engl J Med. 2011;365(8):725-733.

8. Grupp SA, et al. Chimeric antigen receptormodified $\mathrm{T}$ cells for acute lymphoid leukemia. N Engl J Med. 2013;368(16):1509-1518.

9. Brentjens RJ, et al. CD19-targeted T cells rapidly induce molecular remissions in adults with chemotherapy-refractory acute lymphoblastic leukemia. Sci Transl Med. 2013;5(177):177ra38.

10. Gill S, June CH. Going viral: chimeric antigen receptor T-cell therapy for hematological malignancies. Immunol Rev. 2015;263(1):68-89.

11. Gill S, Maus MV, Porter DL. Chimeric antigen receptor $\mathrm{T}$ cell therapy: $25 y$ ears in the making. 
Blood Rev. 2016;30(3):157-167.

12. Louis CU, et al. Antitumor activity and longterm fate of chimeric antigen receptor-positive T cells in patients with neuroblastoma. Blood. 2011;118(23):6050-6056.

13. Ahmed N, et al. Human Epidermal Growth Factor Receptor 2 (HER2)-specific chimeric antigen receptor-modified $\mathrm{T}$ cells for the immunotherapy of HER2-positive sarcoma. J Clin Oncol. 2015;33(15):1688-1696.

14. Yang Y. Cancer immunotherapy: harnessing the immune system to battle cancer. J Clin Invest . 2015;125(9):3335-3337.

15. Khalil DN, Smith EL, Brentjens RJ, Wolchok JD. The future of cancer treatment: immunomodulation, CARs and combination immunotherapy. Nat Rev Clin Oncol. 2016;13(5):273-290.

16. Moon EK, et al. Blockade of programmed death 1 augments the ability of human $T$ cells engineered to target NY-ESO- 1 to control tumor growth after adoptive transfer. Clin Cancer Res.
2016;22(2):436-447.

17. Kalos M, et al. $\mathrm{T}$ cells with chimeric antigen receptors have potent antitumor effects and can establish memory in patients with advanced leukemia. Sci Transl Med. 2011;3(95):95ra73.

18. John LB, et al. Anti-PD-1 antibody therapy potently enhances the eradication of established tumors by gene-modified T cells. Clin Cancer Res. 2013;19(20):5636-5646.

19. Cherkassky L, et al. Human CAR T cells with cell-intrinsic PD-1 checkpoint blockade resist tumor-mediated inhibition. JClin Invest. 2016;126(8):3130-3144.

20. Curran MA, et al. Systemic 4-1BB activation induces a novel $\mathrm{T}$ cell phenotype driven by high expression of Eomesodermin. J Exp Med. 2013;210(4):743-755.

21. Kao C, et al. Transcription factor T-bet represses expression of the inhibitory receptor PD- 1 and sustains virus-specific $\mathrm{CD} 8^{+} \mathrm{T}$ cell responses during chronic infection. Nat Immunol.
2011;12(7):663-671.

22. Di Stasi A, et al. Inducible apoptosis as a safety switch for adoptive cell therapy. $N$ Engl JMed. 2011;365(18):1673-1683.

23. Ciceri F, et al. Infusion of suicide-geneengineered donor lymphocytes after family haploidentical haemopoietic stem-cell transplantation for leukaemia (the TK007 trial): a non-randomised phase I-II study. Lancet Oncol. 2009;10(5):489-500.

24. Morgan RA, Yang JC, Kitano M, Dudley ME, Laurencot CM, Rosenberg SA. Case report of a serious adverse event following the administration of $\mathrm{T}$ cells transduced with a chimeric antigen receptor recognizing ERBB2. Mol Ther. 2010;18(4):843-851.

25. Kloss CC, Condomines M, Cartellieri M, Bachmann M, Sadelain M. Combinatorial antigen recognition with balanced signaling promotes selective tumor eradication by engineered $\mathrm{T}$ cells. Nat Biotechnol. 2013;31(1):71-75. 